\title{
Risk Forecasting of Karachi Stock Exchange: A Comparison of Classical and Bayesian GARCH Models
}

\author{
Farhat Iqbal \\ Department of Statistics, University of Balochistan \\ Quetta-Pakistan \\ farhatiqb@gmail.com
}

\begin{abstract}
This paper is concerned with the estimation, forecasting and evaluation of Value-at-Risk (VaR) of Karachi Stock Exchange before and after the global financial crisis of 2008 using Bayesian method. The generalized autoregressive conditional heteroscedastic $(\mathrm{GARCH})$ models under the assumption of normal and heavy-tailed errors are used to forecast one-day-ahead risk estimates. Various measures and backtesting methods are employed to evaluate VaR forecasts. The observed number of VaR violations using Bayesian method is found close to the expected number of violations. The losses are also found smaller than the competing Maximum Likelihood method. The results showed that the Bayesian method produce accurate and reliable $\mathrm{VaR}$ forecasts and can be preferred over other methods.
\end{abstract}

Keywords: GARCH, Volatility, Value-at-Risk, MCMC.

\section{Introduction}

Value-at-Risk (VaR) has become a popular tool and is widely used for risk management and capital allocation by financial institutions. Both underestimation and overestimation of risk could have a negative effect in financial markets. Therefore accurate estimates of $\mathrm{VaR}$ is crucial for the financial stability of markets. VaR can be defined as the quantile of the loss, during a specific time period, that can occur within a given portfolio. A precise quantile estimate far out in the left tail of the return distribution is desirable. A thorough survey of risk measures is provided by Jorion (2007).

Various approaches for estimating and predicting VaR exist in literature. These include nonparametric method such as historical simulation; semi-parametric method based on extreme value theory and quantile regression method and full parametric models (see McNeil and Frey, 2000 and Engle and Manganelli, 2004, among others). Kuester et al. (2006) provided an overview and comparisons of these and further methods whereas a comprehensive overview is found in Abad et al. (2014).

Under the parametric statistical approaches, the autoregressive conditional heteroscedastic (ARCH) model of Engle (1982) and generalized ARCH (GARCH) model of Bollerslev (1986) are widely-used by researchers and practitioners. These models can capture the conditional variance structure and some of the stylized facts of many financial time series. Since then numerous extensions of the GARCH model have been proposed. Among them, the exponential GARCH model of Nelson (1991) and asymmetric model of Glosten et al. (1993) are popular. Accurate volatility estimates are essential for producing reliable VaR estimates.

The estimates of the parameters of GARCH model is usually obtained using the Gaussian maximum likelihood and the estimator is often called the quasi maximum likelihood 
estimator (QMLE). However, normality is often rejected in applications as the unconditional distribution of most financial asset returns has fatter tails than implied by this model with normal errors. The excess of (unconditional) kurtosis has been most commonly accommodated with Student-t distributed errors (e.g. Baillie and Bollerslev, 1989). Besides, robust methods for the estimation of GARCH models are also suggested (Peng and Yao, 2003; Muller and Yohai, 2008; Iqbal and Mukherjee, 2010 to cite few)

Another approach to estimate the parameters and volatility of GARCH model is to use Bayesian framework. The Bayesian paradigm offers a natural way of taking both parameter uncertainty and model uncertainty into account. However, the literature on Bayesian treatment of GARCH model in not as enormous as in the case of QMLE. In Bayesian setup, most of the time a researcher has to rely on computational methods such as Markov Chain Monte Carlo (MCMC) for the estimation of these models (see Nakatsuma, 1998, 2000, Ardia, 2008 and Deschamps, 2012).

Karachi Stock Exchange (KSE) is the major stock market of Pakistan. Most of the studies on risk estimated of KSE that exist in literature applied classical methods for estimation and prediction. Iqbal et al. (2010) used four different parametric methods and two nonparametric methods for VaR computation of KSE. Qayyum and Nawaz (2010) used extreme value theory and Nawaz and Afzal (2011) computed the VaR using Historical Simulation and Risk Metrics method. Mahmud and Mirza (2011) forecast the volatility of KSE before and after the financial crisis using GARCH models. Haque and Naeem (2014) investigated the volatility forecasting performance of GARCH models with various distribution of innovations. To the best of our knowledge, the Bayesian framework has not been yet applied for volatility or risk forecasting of KSE. This motivate us to fill this gap and contribute to the literature.

The main aim of this paper is to estimate and forecast VaR using GARCH models in Bayesian setup. The QMLE method is also used for the comparison of results. The estimates and forecasts of volatility and $\mathrm{VaR}$ are computed by these two methods using Gaussian and Student-t distributions for innovations. Various evaluation measures and backtesting methods are employed to compare the in-sample and out-of-sample forecasts of VaR. The daily closing prices of KSE from January 03, 2005 to December 30, 2011 are used in the present study. This study is important from various angles. First, as aforementioned, the VaR estimates of KSE are calculates using Bayesian method that to the best of our knowledge has not been studied. Another contribution of this study is to check the effects of large shocks on risk estimated of KSE not only in the full time period but also before and after the global financial crisis. This may help practitioners and researchers to understand the behaviour of risk measure when the market is hit by large shocks at different time periods and their after effects. Finally, the use of Bayesian method for forecasting in GARCH model may encourage academicians and researchers in Pakistan to apply Bayesian methods for local financial data.

The rest of the paper is organized as follows: In Section 2, a brief overview of GARCH model and both estimation methods are presented. Value-at-Risk estimation and prediction are briefly discussed along with various evaluation measures in Section 3. In Section 4, the results of this study are reported and discussed. Finally, Section 5 concludes the article. 


\section{GARCH Model and Estimation}

For the simple GARCH $(1,1)$ model, the following representation of the return series $\left\{y_{t} ; t \in \mathbb{Z}\right\}$ is assumed. Observer $\left\{y_{t} ; 1 \leq t \leq T\right\}$ such that

$$
\begin{aligned}
& y_{t}=h_{t}^{1 / 2} \epsilon_{t}, \quad \epsilon_{t} \sim D(0,1) \\
& h_{t}=\omega_{0}+\alpha_{0} y_{t-1}^{2}+\beta_{0} h_{t-1},
\end{aligned}
$$

where $\left\{\epsilon_{t}\right\}$ is a sequence of independent and identically distributed (i.i.d.) unobservable real-valued random variables with mean 0 and variance 1 and distribution $D$; and $\boldsymbol{\theta}_{\mathbf{0}}=\left[\omega_{0}, \alpha_{0}, \beta_{0}\right]^{\prime}$, the unknown parameter vector in the parameter space

$$
\boldsymbol{\Theta}=\left\{\boldsymbol{\theta}=[\omega, \alpha, \beta]^{\prime} ; \omega>0, \alpha, \beta \geq 0,(\alpha+\beta)<1\right\} .
$$

Under these parameter constratints, The $\operatorname{GARCH}(1,1)$ model in $(2.1)$ is strictly stationary and hence covariance stationary under finite second moment.

In this study, two error distributions are used for the i.i.d innovations. The choice $D(0,1) \equiv N(0,1)$ is a standard and Student-t distribution. Th later is standardized to have zero mean and unit variance.

The conditional likelihood can be written as:

$$
L_{T}(\boldsymbol{y} \mid \boldsymbol{\theta})=\prod_{t=1}^{T} \frac{1}{\sqrt{h_{t}}} f_{\epsilon}\left(\frac{y_{t}}{\sqrt{h_{t}}}\right),
$$

where $\boldsymbol{y}=\left(y_{1}, \cdots, y_{T}\right)^{\prime}$ and $f_{\epsilon}$ is the relevant error density function of $\epsilon_{t}$. In the classical setup, $\boldsymbol{\theta}$ is assumed to be the true and fixed value and the maximum likelihood estimator of $\boldsymbol{\theta}$ is then obtained by

$$
\widehat{\boldsymbol{\theta}}_{T}=\arg \max _{\boldsymbol{\Theta}} L_{T}(\boldsymbol{\theta}) \text {. }
$$

In the Bayesian setup, $\boldsymbol{\theta}$ is considered to be a random variable with a prior density $p(\boldsymbol{\theta})$ which depends on the researcher's prior belief. These parameters are assumed to be a priori independent and normally distributed truncated to the intervals that define each one. It is also assumed that few known and constant hyperparameters specify their densities. For example, as proposed in Ardia (2008), these are given by $\omega \sim N\left(\mu_{\omega}, \sigma_{\omega}^{2}\right) I_{(\omega>0)}, \alpha \sim N\left(\mu_{\alpha}, \sigma_{\alpha}^{2}\right) I_{(0<\alpha<1)}$ and $\beta \sim N\left(\mu_{\beta}, \sigma_{\beta}^{2}\right) I_{(0<\beta<1)}$, where $I$ is the indicator function.

The posterior density of $\boldsymbol{\theta}$ is obtained using the Bayes' rule as

$$
p(\boldsymbol{\theta} \mid \boldsymbol{y})=\frac{L(\boldsymbol{\theta} \mid \boldsymbol{y}) p(\boldsymbol{\theta})}{\int_{\boldsymbol{\Theta}} L(\boldsymbol{\theta} \mid \boldsymbol{y}) p(\boldsymbol{\theta}) \mathrm{d} \boldsymbol{\theta}},
$$

where $L(\boldsymbol{\theta} \mid \boldsymbol{y}) \equiv p(\boldsymbol{y} \mid \boldsymbol{\theta})$ is the likelihood function of the model parameters and the denominator is the so called normalizing constant. But, this posterior distribution is generally not anlaytically tractable. Therefore, MCMC sampling strategies are adopted for obtaining samples from the joint posterior distributions. These include the famous 
Metropolis-Hasting algorithm (Metropolis et al., 1953 and Hasting, 1970) and the Gibbs sampler (Geman and Geman, 1984), among others.

For the GARCH $(1,1)$ model in $(2.1)$ and assuming $\epsilon_{t} \sim N(0,1)$, the likelihood function of $\boldsymbol{\theta}$ can be written as:

$$
L(\boldsymbol{\theta} \mid \boldsymbol{y}) \propto(\operatorname{det} \boldsymbol{\Sigma})^{-\frac{1}{2}} \exp \left[-\frac{1}{2} \boldsymbol{y}^{\prime} \boldsymbol{\Sigma}^{-1} \boldsymbol{y}\right],
$$

where $\boldsymbol{\Sigma}:=\boldsymbol{\Sigma}(\boldsymbol{\theta})=\operatorname{diag}\left(\left\{h_{t}(\boldsymbol{\theta})\right\}_{t=1}^{T}\right)$ and det is the determinant of a matrix. The likelihood for Student- $t$ distribution can also be written in a similar fashion with additional parameter, $v$, the degress of freedom, that is also estimated with the parameters. For a comprehensive details of MCMC method in GARCH model, interested reader are referred to Nakatsuma (1998) and Ardia (2008).

\section{Value-at-Risk Forecasting and Evaluation}

This section first describes the method of predicting VaR using GARCH model. Then, evaluation measures and bactesting methods are presented to evaluate these VaR estimates. VaR measures the worst expected loss of a portfolio over a target horizon at a given confidence level, due to an adverse movement in the relevant security price (Jorion, 2007). For a known probability $p$, a $(1-p) 100 \% \mathrm{VaR}$ is defined as the $p$ th conditional quantile of the returns. Hence the $\operatorname{VaR}$ at time $t>1$, for the returns $\left\{y_{t} ; 1 \leq t \leq T\right\}$ is defined as

$$
q_{t}=\inf \left\{y ; p \leq P_{t-1}\left(y_{t} \leq y\right)\right\}
$$

where $P_{t-1}$ is the conditional distribution of $y_{t}$ given then information available up to $t-1$. Hence, from (2.1) we get $q_{t}=h_{t}^{1 / 2}\left(\boldsymbol{\theta}_{0}\right) F^{-1}(p)$ where $F^{-1}$ is the quantile function of the innovation $\left\{\epsilon_{t}\right\}$. The estimate of $\mathrm{VaR}$ is then defined as

$$
\hat{q}_{t}=\hat{h}_{t}^{1 / 2}\left(\widehat{\boldsymbol{\theta}}_{T}\right) \times([T p]+1) \text { th order statistics of }\left\{y_{t} / \hat{h}_{t}^{1 / 2}\left(\widehat{\boldsymbol{\theta}}_{T}\right)\right\}, 2 \leq t \leq T
$$

The Basel Committee on Banking Supervision (1996) recommends a backtesting procedure to evaluate the accuracy of $\mathrm{VaR}$ forecasts. This is generally based on the number of observed violations, i.e. when actual losses exceed VaR in a sample period.

\subsection{Coverage probability and violation rate}

Let us define the total number of observed violations as $T_{*}$

$$
T_{*}=\sum_{t=2}^{T} I_{t} \quad \text { with } \quad I_{t}=I\left(y_{t} \leq \hat{q}_{t}\right)
$$

Then the closeness of empirical rejection probability $\hat{p}=T_{*} / T$ to ' $p$ ' can be used to assess the overall predicative performance of the $\mathrm{VaR}$ model. This probability also known as $\mathrm{VaR}$ violation rate provides an interesting insight to $\mathrm{VaR}$ forecasts.

In case a model correctly specifies the conditonal quantile of returns, its true $\hat{p}$ should be equal to nominal level $p$. The ratio $\hat{p} / p$ can be used to compare and rank competing 
models. Of course, a model with this ratio close to unity is prefered and in case of ties, conservative model $(\hat{p} / p<1)$ is chosen as superior .

\subsection{Average quadratic loss}

The magnitude of losses is also important in the evaluation of VaR. Lopez (1999) considered this magnitude and defined the average quadratic loss (AQL) of a VaR estimate. The overall AQL of a VaR estimate is obtained as $\sum_{t=1}^{T} A Q L_{t} / T$, where

$$
A Q L_{T}=\left\{\begin{array}{lll}
1+\left(\hat{q}_{t}-y_{t}\right)^{2} & \text { if } y_{t} \leq \hat{q}_{t} \\
0 & \text { if } y_{t}>\hat{q}_{t}
\end{array}\right.
$$

Next, we define formal tests for backtesting VaR estimates.

\subsection{Coverage tests}

The first test is the unconditional likelihood ratio test proposed by Kupiec (1995). It is defined as

$$
\mathrm{LR}_{\mathrm{uc}}=2\left[\ln \left\{(1-\hat{p})^{T-T_{*}} \hat{p}^{T_{*}}\right\}-\ln \left\{(1-p)^{T-T_{*}} p^{T_{*}}\right\}\right]
$$

which is asymptotically $\chi_{(1)}^{2}$.

Christoffersen (1998) defined the independence coverage test statistic, denoted by $\mathrm{LR}_{\text {ind }}$ as follows. For $i, j=0,1$, let $T_{i j}$ denotes the number of time points $\{t ; 2 \leq t \leq T\}$ for which $I_{t}=i$ is followed by $I_{t+1}=j$. Let

$$
\hat{\pi}_{i j}=T_{i j} /\left(T_{i 0}+T_{i 1}\right), \quad \widehat{\pi}=\left(T_{01}+T_{11}\right) / T
$$

Then

$$
\mathrm{LR}_{\text {ind }}=2\left[\ln \left(\left(1-\hat{\pi}_{01}\right)^{T_{00}} \hat{\pi}_{01}^{T_{01}}\left(1-\hat{\pi}_{11}\right)^{T_{10}} \hat{\pi}_{11}^{T_{11}}\right)-\ln \left((1-\hat{\pi})^{\left(T_{00}+T_{10}\right)} \hat{\pi}^{\left(T_{01}+T_{11}\right)}\right)\right]
$$

The conditional coverage test statistic of Christoferssen (1998) which is asymptotically $\chi_{(2)}^{2}$ is

$$
\mathrm{LR}_{\mathrm{cc}}=\mathrm{LR}_{\mathrm{uc}}+\mathrm{LR}_{\mathrm{ind}}
$$

\subsection{Dynamic quantile test}

Higher order dependence in $\mathrm{VaR}$ violations also need to be checked. The dynamic quantile (DQ) test of Engle and Manganelli (2004) is used for this purpose. This test is described as follows. Let the $t$ th response $h_{t}, 2 \leq t \leq T$ be

$$
h_{t}=\left\{\begin{array}{cl}
1-p & \text { if } X_{t} \leq \hat{q}_{t} \\
-p & \text { if } X_{T}>\hat{q}_{t}
\end{array}\right.
$$

and $h_{1}=-p$. Consider a linear regression model with response $\boldsymbol{Y}=\left[h_{1}, \cdots, h_{T}\right]^{\prime}$ and a $T \times k$ design matrix $\boldsymbol{X}=\left[x_{t, j}\right]$ with $k=4$ and all ones in the first column. For the $(t, j)$ th term with $2 \leq j \leq 3, x_{t, j}=h_{t-j}$ if $j<t$ and $x_{t, j}=0$ if $j \geq t$ and $x_{t, 4}=\hat{q}_{t}$. The DQ test statistic is asymptotically $\chi_{(4)}^{2}$ and is defined as

$$
\mathrm{DQ}=\frac{\widehat{\boldsymbol{\beta}}^{\prime} \boldsymbol{X}^{\prime} \boldsymbol{X} \widehat{\boldsymbol{\beta}}}{p(1-p)}
$$

Where $\widehat{\boldsymbol{\beta}}=\left(\boldsymbol{X}^{\prime} \boldsymbol{X}\right)^{-1}\left(\boldsymbol{X}^{\prime} \boldsymbol{Y}\right)$ is the ordinary least square estimator. 
These measures and tests are used in this study to evaluate and compare the performance of risk estimates of QMLE and Bayesian method.

\section{Empirical Results and Discussion}

\subsection{Data and preliminary analysis}

The present study uses the daily closing prices of Karachi Stock Exchange (KSE 100 Index). The dataset is obtained from the http://finance.yahoo.com for the period of January 03, 2005 to December 31, 2011. This period include the high volatile period because of the global financial crisis. This may help us to understand the dynamics of KSE before and after the financial crisis. The full data set consists of 1686 observations. The data is later divided into two periods: the pre-crisis period (03 January $2005-30$ August 2008) consisting of 907 observations, and the post-crisis period (01 September 2008 - 31 December 2011) having 779 data points. The KSE was almost static for few weeks during the last quarter of 2008 and therefore few observations (from 09 October 2008 - 12 December 2008) are removed.

The returns at time $t$ is defined as $r_{t}=\ln \left(P_{t} / P_{t-1}\right) \times 100 \%$, for $t=1, \ldots, T$, where $P_{t}$ is the closing index of KSE at time $t$. Then using $\left\{y_{t}=r_{t}-\bar{r}_{t} ; 1 \leq t \leq T\right\}$ (with $\left.\bar{r}_{t}=\sum_{t=1}^{T} r_{t} / T\right)$ as our observations, the whole span in each time period is divided into two parts: the estimation or in-sample part of initial $K$ observations used for estimating the unknown parameters in GARCH models and the validation or out-of-sample part of $N=T-K$ observations for the prediction and assessment of VaR. For out-of-sample forecasting, $N=250$ which corresponds to one year observations. The recommended back-testing guideline proposed by the Basel Committee on Banking Supervision (1996) is to also evaluate a one percent (1\%) VaR model over a 12 month test period (250 trading days). The daily closing prices (in thousands US dollar) and log-returns of KSE are shown in Figure 1 below. The effect of global financial crisis is evident on KSE. A large drop in the prices and high volatility and volatility clustering can also be seen in in log-returns.
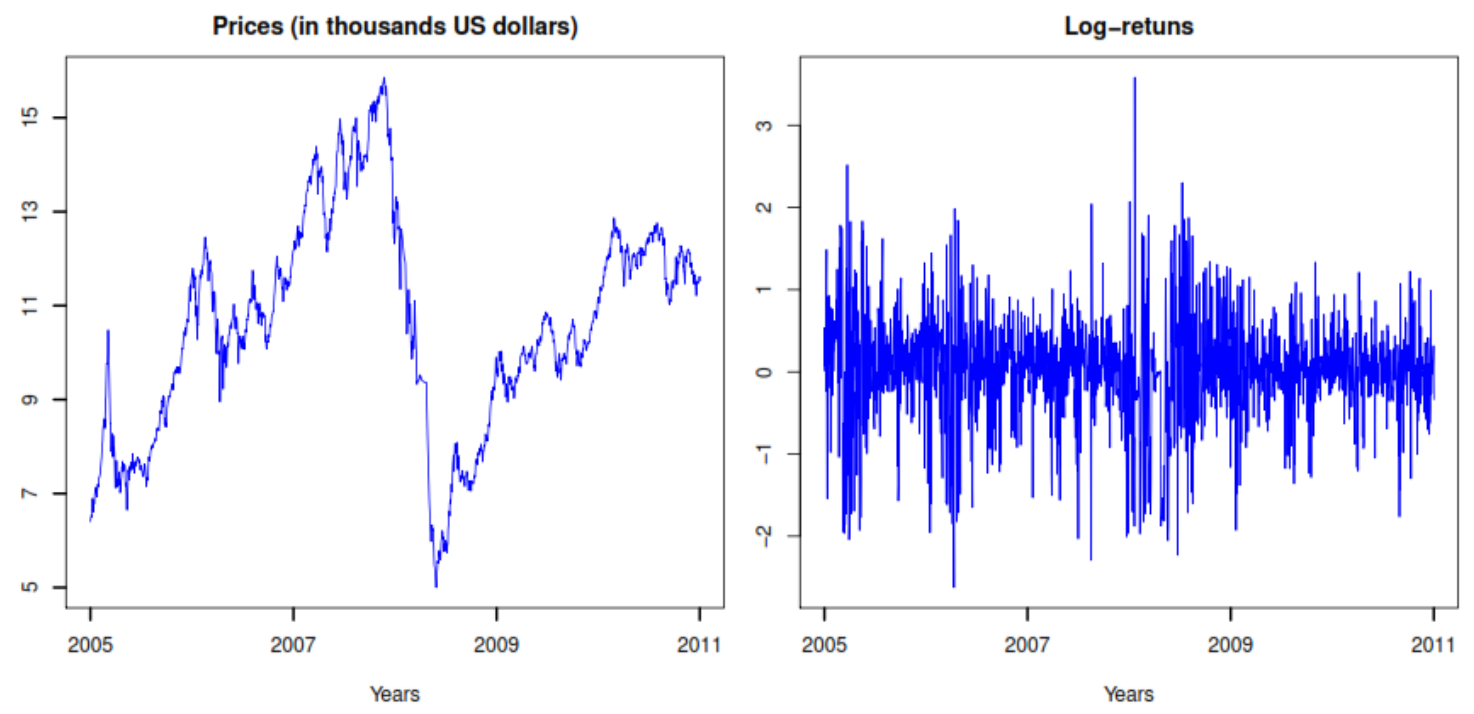

Figure 1. Daily closing prices (left) and log-returns (right) of KSE 
Table 1 presents the summary statistics for the full, before the crisis and after the crisis time periods. These statistics may help us to examine the behaviour of the stock returns. All time periods have higher kurtosis and negative skewness in returns. The normality in returns was tested using Jarque-Bera test for normality and high values of this test show that the KSE returns are significantly different from normality. High values of Ljung-Box $\left(Q^{2}\right)$ statistic for the squared returns at lag 20 were also observed in all time periods. This is the indication of dependence in squared returns and thus a need for fitting GARCH models. In summary, the KSE return series do not conform to normal distribution, display negative skewness (the distribution has a long left tail) and high kurtosis (fat tails) for all periods. It can also be noticed that the unconditional volatility is higher just before the crisis period.

Table 1: Summary statistics for daily return of Karachi Stock Exchange

\begin{tabular}{llll}
\hline Periods & Full & Before crisis & After crisis \\
\hline Sample size & 1686 & 907 & 779 \\
Mean & 0.0145. & 0.0169 & 0.0117 \\
Median & 0.0476 & 0.0828 & 0.0149 \\
Minimum & -2.6240 & -2.2640 & -2.2300 \\
Maximum & 3.5850 & 3.5850 & 2.3020 \\
SD & 0.6158 & 0.8755 & 0.5884 \\
Skewness & -0.3385 & -0.3599 & -0.2778 \\
Kurtosis & 4.8973 & 4.5592 & 5.0183 \\
JB & 285.08 & 111.46 & 142.24 \\
$Q^{2}(20)$ & 1656.90 & 782.44 & 897.81 \\
\hline
\end{tabular}

Note: Full period (03 January 2005 - 31 December 2011); Before crisis period (03 January 2005 - 30 August 2008); After crisis period (01 September 2008 - 31 December 2011); JB (Jarque-Bera statistic for normality of return); $Q^{2}$ (Ljung-Box statistics at lag 20 for serial correlation in squared returns).

Next, the GARCH model is fitted to all three time periods. The in-sample estimates of parameters, volatility and VaR are obtained using the QMLE and Bayesian method under the assumptions of both Gaussian and Student-t distributions. For the MCMC method, a total of 10,000 iterations are run discarding the first 2,000 realizations as burn-in period. Posterior results are then based on 8,000 realizations of the Markov chain with the prior distributions as explained in Section 2. The simulated Markov chains are checked for convergence and good mixing by visual inspection of the marginal traces, density estimates, and autocorrelations are observed. Figure 2 shows an illustration of the diagnostic plots.

\subsection{In-sample VaR analysis}

Table 2 presents the in-sample VaR results of both estimation methods. First, the results of QMLE and Bayesian methods under Gaussian assumption for errors are discussed. For the sake of brevity, the results of only $p=0.10$ (90\% VaR confidence level) is presented. Similar results are obtained for other levels. As it can be seen that both 
methods produce reasobaly indentical results for the full time period. The observed ratios $\hat{p} / p$ were found close to 1 which means that the number of observed violations are close to expected number of violations. The results of all three tests (unconditional and conditional coverage tests and dynamic quantile test) values divided by their respective critical values at 5\% confidence level are also reported. A value greater than 1 means rejection of the test at 5\% level. Non-significant values for unconditional coverage test and significant values for conditional coverage and DQ test are found using QMLE. In Bayesian method, again only unconditional test is found non-significant. The average quadratic loss (AQL) produced by both methods are similar in magnitude.

For other two time periods (before and after financial crisis) considered in this study, some interesting findings are noticed. The expected and observed number of violation in Bayesian showed exactly same values, therefore VaR ratios of 1 can be seen in these periods. The number of observed violation for QMLE are found smaller than the expected violations. In after crisis period the conditional coverage and DQ tests are also rejected by QMLE whereas non-significant values of these test statistics in Bayesian confirms independence of violations from their lags and past VaR. Finally, the AQL of Bayesian is also found smaller than the QMLE in both time periods meaning that the losses using Bayesian are less on average.
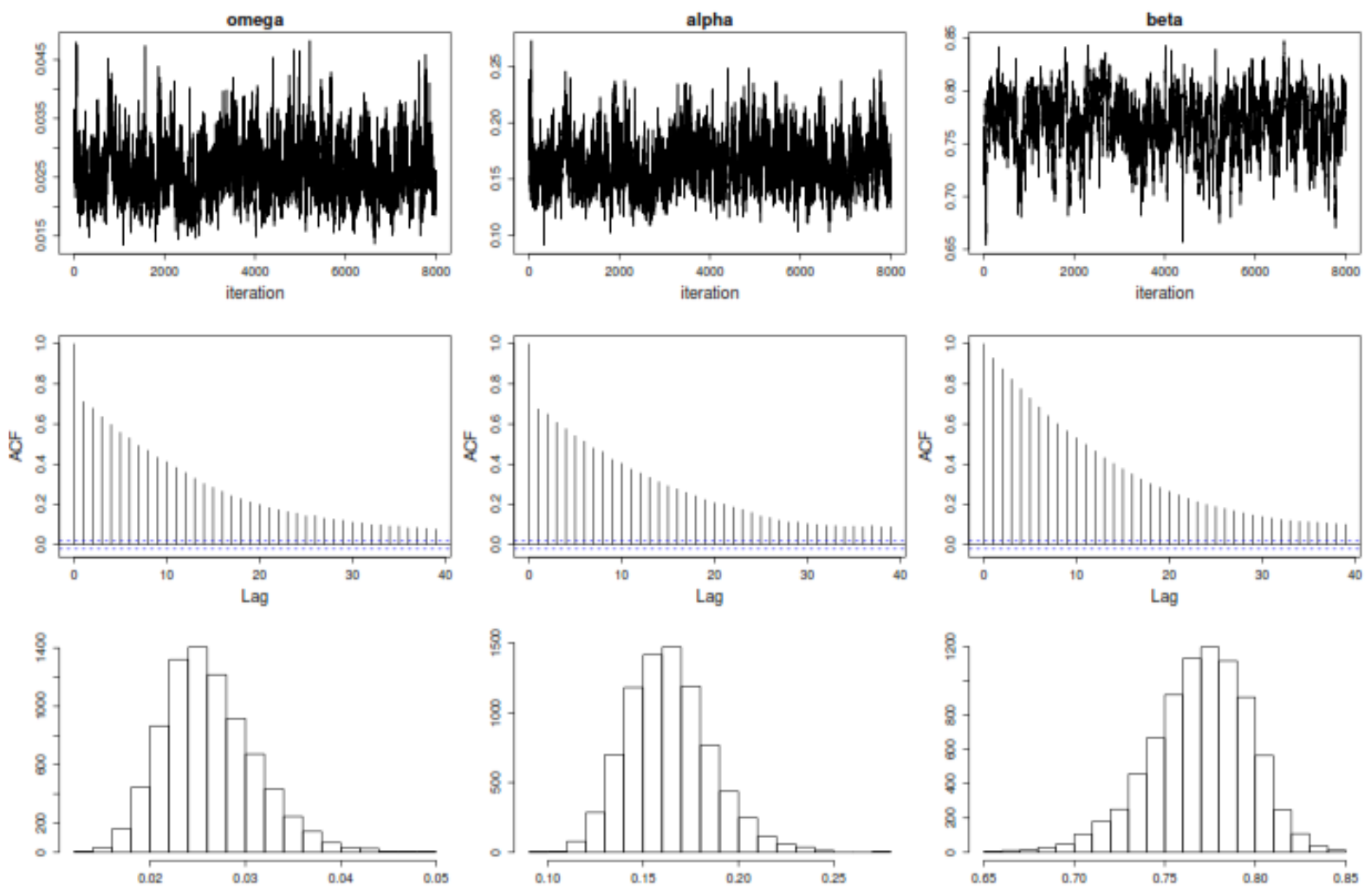

Figure 2. Trace plots (top), autocorrelations (middle) and histograms (bottom) of parameters 
The result of in-sample VaR when Student-t distribution was assumed for errors showed similar trend. With Bayesian method outperforming the QMLE in terms of better VaR ratios, nonsignificant test statistics and lower AQL. Another feature that can be observed is that these results get slightly better than those in Gaussian case. This indicates that Student-t distribution is preferred for better risk forecasting in GARCH models. In summary, the Bayesian method provides reliable VaR forecasts than the QMLE and further improves the forecasts when heavy-tailed distribution is assumed for errors.

\subsection{Out-sample VaR analysis}

For policy and risk management, prediction and evaluation of $\mathrm{VaR}$ are considered more important. This subsection highlights the results of out-of-sample VaR forecats. To produce one-day-ahead forecast of $\mathrm{VaR}$, a rolling window approach is used. More specifically, the model is fitted to the estimation period using in-sample part of $K$ observations and one-day-ahead forecasts are obtained. Then the in-sample period is rolled forward by one day dropping the first observation. The model is re-estimated and again the next day forecasts are obtained. In this way out-of-sample VaR forecasts of approximately one year (250 days) are obtained for the forecast period using both methods.

Table 3 reports the results of $\mathrm{VaR}$ forecasts. Starting with the number of expected and observed voilations, it is found that though QMLE produced VaR ratios close to 1 in full and pre crisis period, the higher value of $\hat{p} / p$ in after crisis period was observed. This is an indication that the QMLE understimated the market risk by producing number of voilations largert than the expected number of violation in a specific period. Understimated VaR is considered more risky than the overstimated VaR. All three tests are also rejected in this time period and average losses (AQL) are also on the higher side in case of QMLE. The Bayesian method again performed reasonbly well in predicting one-step-ahead VaR. In all time periods, the observed number of violations are found close to the expected numbers with smaller losses. Hence, it is conlcuded that this method provide better forecast of risk under different market conditions. 
Table 2: In-sample VaR evaluation using GARCH model

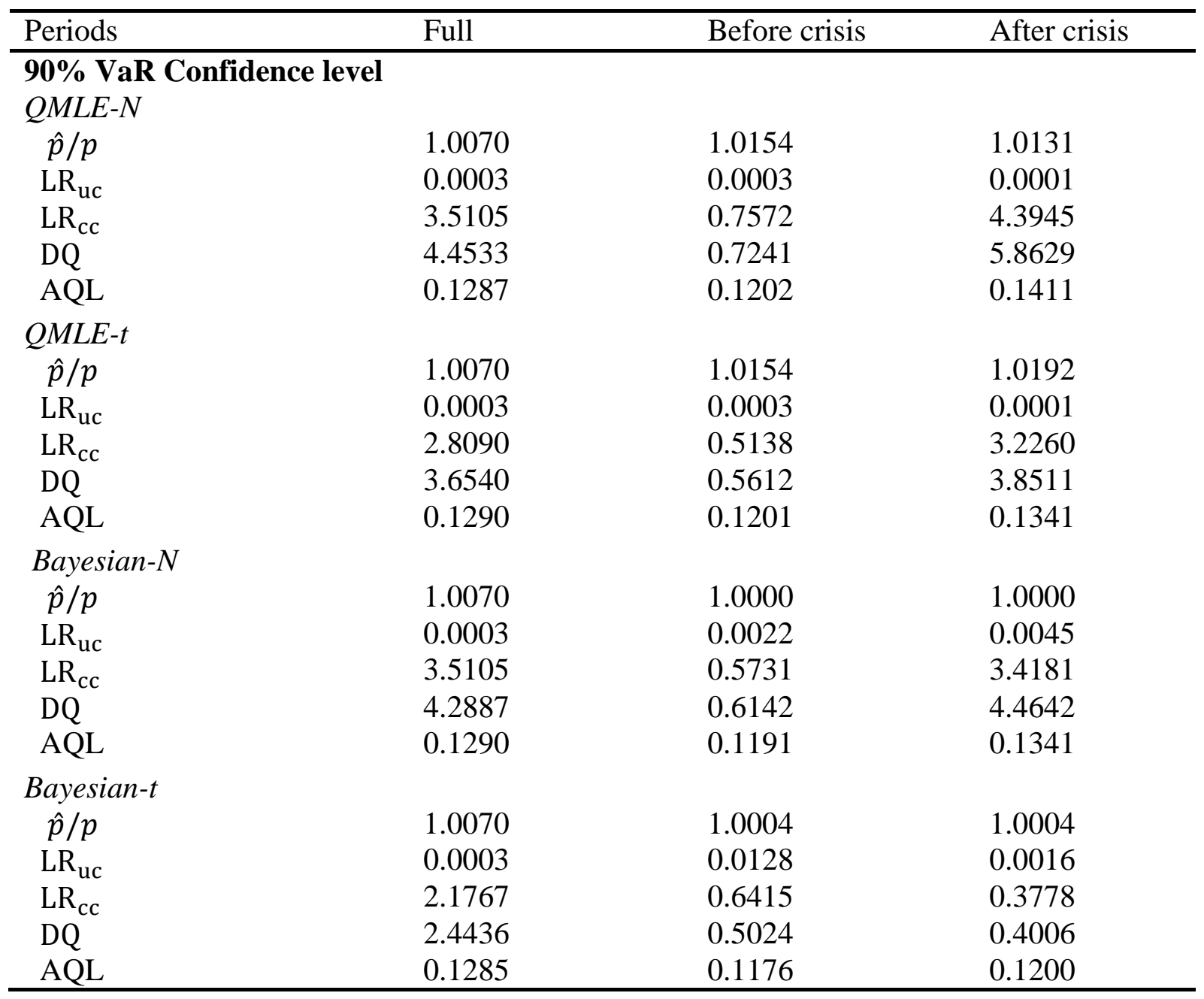

Note: Full period (03 January 2005 - 31 December 2011); Before crisis period (03 January 2005 - 30 August 2007); After crisis period (01 September 2008 - 31 December 2013); DQ (Dynamic quantile statistic); AQL (Average quadratic loss); the smallest AQL is in bold type; For all three tests, >1 means rejection at $5 \%$ level.

\section{Conclusion}

Maximum likelihood is a popular and widely-used method for the estimation of GARCH models. Bayesian methods can also provide a reasonably good estimates for GARCH models. In this paper, both these methods are fitted to the daily data of Karachi Stock Exchange returns before and after the global financial crisis. The estimates of in-sample and out-of-sample VaR are computed. Various evaluation measures and backtesting methods are applied to assess the accuracy of VaR forecast for both estimation methods. The result of the study showed that the Bayesian method can be safely used for the prediction of $\mathrm{VaR}$ as it provides reasonable $\mathrm{VaR}$ ratios and smaller losses. Bayesian methods in finance have gained a lot of attention of researchers. In Pakistan, this area of research is still lacking. The findings of this study may encourage researchers and academicians in Pakistan to adopt Bayesian paradigm for volatility and risk analysis of financial variables. 
This study can be extended in following ways: The MCMC method used is very time consuming. New and efficient Bayesian methods such as Sequential Monte Carlo can be used for online estimation and prediction of GARCH models. A simple GARCH model is studied whereas other variants that consider asymmetry and jumps in volatility can be considered and may provide better risk forecasts.

Table 3: Out-of-sample VaR evaluation using GARCH model

\begin{tabular}{|c|c|c|c|}
\hline Periods & Full & Before crisis & After crisis \\
\hline \multicolumn{4}{|c|}{ 90\% VaR Confidence level } \\
\hline \multicolumn{4}{|c|}{$Q M L E-N$} \\
\hline$\hat{p} / p$ & 1.0000 & 0.8400 & 1.4800 \\
\hline $\mathrm{LR}_{\mathrm{uc}}$ & 0.0000 & 0.1947 & 1.4741 \\
\hline $\mathrm{LR}_{\mathrm{cc}}$ & 0.0150 & 0.1305 & 1.2190 \\
\hline $\mathrm{DQ}$ & 0.7059 & 0.3611 & 1.6112 \\
\hline AQL & 0.1252 & 0.1513 & 0.1849 \\
\hline \multicolumn{4}{|l|}{$Q M L E-t$} \\
\hline$\hat{p} / p$ & 1.1200 & 0.8400 & 1.9200 \\
\hline $\mathrm{LR}_{\mathrm{uc}}$ & 0.1006 & 0.1947 & 4.9613 \\
\hline $\mathrm{LR}_{\mathrm{cc}}$ & 0.0646 & 0.1305 & 3.2053 \\
\hline DQ & 0.6790 & 0.3634 & 3.0394 \\
\hline AQL & 0.1421 & 0.1481 & 0.2347 \\
\hline \multicolumn{4}{|c|}{ Bayesian- $N$} \\
\hline$\hat{p} / p$ & 0.8400 & 1.0000 & 1.2000 \\
\hline $\mathrm{LR}_{\mathrm{uc}}$ & 0.1946 & 0.0000 & 0.2736 \\
\hline $\mathrm{LR}_{\mathrm{cc}}$ & 0.1882 & 0.1603 & 0.1907 \\
\hline $\mathrm{DQ}$ & 0.5256 & 0.4047 & 1.0139 \\
\hline AQL & 0.1010 & 0.1455 & 0.1476 \\
\hline \multicolumn{4}{|c|}{ Bayesian- $t$} \\
\hline$\hat{p} / p$ & 0.9200 & 0.1004 & 0.9600 \\
\hline $\mathrm{LR}_{\mathrm{uc}}$ & 0.0474 & 0.0000 & 0.2131 \\
\hline $\mathrm{LR}_{\mathrm{cc}}$ & 0.1562 & 0.1604 & 0.1441 \\
\hline $\mathrm{DQ}$ & 0.3848 & 0.4059 & 0.3983 \\
\hline $\mathrm{AQL}$ & 0.1103 & 0.1447 & 0.1454 \\
\hline
\end{tabular}

Note: Full period (03 January 2005 - 31 December 2011); Before crisis period (03 January 2005 - 30 August 2007); After crisis period (01 September 2008 - 31 December 2013); DQ (Dynamic quantile statistic); AQL (Average quadratic loss); the smallest AQL is in bold type; For all three tests, $>1$ means rejection at $5 \%$ level.

\section{References}

1. Abad, P., Benito, S. and Lopez, C. (2014). A comprehensive review of Value at Risk methodologies. The Spanish Review of Financial Economics. 12: 15 - 32.

2. Ardia., D. (2008). Financial Risk Management with Bayesian Estimation of GARCH Models: Theory and Applications. Springer-Verlag, Berlin. 
3. Baillie, R. and Bollerslev, T. (1989). The message in daily exchange rates: A conditional-variance tale. Journal of Business and Economic Statistics. 7(3): 297305.

4. Basel Committee on Banking Supervision. (1996). Supervisory framework for the use of Backtesting in conjunction with the international model-based approach to market risk capital requirements. BIS, Basel, Switzerland.

5. Bollerslev, T. (1986). Generalised autoregressive conditional heteroscedasticity. Journal of Econometrics. 31(3): 307 - 327.

6. Christoffersen, P. (1998). Evaluating interval forecasts. International Economics Review. 39: 841-862.

7. Deschamps, J.P. (2012). Bayesian estimation of generalized hyperbolic skewed student GARCH models. Computational Statistics \& Data Analysis. 56(11): 3035-3054.

8. Engle, R.F. (1982). Autoregressive Conditional Heteroskedasticity With Estimates of the Variance of U.K. Inflation. Econometrica. 50: 987 - 1008.

9. Engle, R.F. and Manganelli, S. (2004) CAViaR: conditional autoregressive value at risk by regression quantiles. Journal of Business and Economic Statistics. 22: 367-381.

10. Geman, S. and Geman, D. (1984). Stochastic relaxation, Gibbs distributions, and the Bayesian restoration of images. IEEE Transactions on Pattern Analysis and Machine Intelligence. 6: 721 - 741.

11. Glosten, L. Jagannathan, R. and Runkle, D. (1993). On the relation between the expected value and the volatility on the nominal excess return on stocks. Journal of Finance. 48(5): 1779 - 1801.

12. Hastings, W. (1970). Monte Carlo sampling methods using Markov chains and their applications. Biometrika. 57: 97-109.

13. Haque, A. and Naeem, K. (2014). Forecasting volatility and Value-at-Risk of Karachi Stock Exchange 100 Index: Comparing distribution-type and symmetrytype models. European Online Journal of Natural and Social Sciences. 3(2): 208219.

14. Iqbal, J., Azher, S. and Ijaz, A. (2010). Predictive ability of Value-at-Risk methods: evidence from the Karachi Stock Exchange-100 Index. MPRA Paper 01/2010, University Library of Munich, Germany.

15. Iqbal, F. and Mukherjee, K. (2010). M-estimators of some GARCH-type models; computation and application. Statistics and Computing. 20: $435-445$.

16. Jorion, P. (2007). Value at Risk: The New Benchmark for Managing Financial Risk. 3rd edn, McGraw-Hill, New York.

17. Kuester K., Mittinik, S. and Paolella M. (2006). Value-at-risk prediction: a comparison of alternative strategies. Journal of Financial Econometrics. 4: 53-89. 
18. Kupiec, P.H. (1995) Techniques for verifying the accuracy of risk measurement models. Journal of Derivatives. 3: 73-84.

19. Lopez, J.A. (1999). Methods for evaluating value-at-risk estimates. Economic Review. 2: 3-17.

20. Mahmud, M. and Nawazish, M. (2011). Volatility and dynamics in an emerging economy: Case of Karachi Stock Exchange. Ekonomska Istraživanja. 24(4): $51-64$.

21. Metropolis, N., Rosenbluth, A., Rosenbluth, M., Teller, A. and Teller, E. (1953). Equations of state calculations by fast computing machines. Journal of Chemical Physics. 21: 1087-1091.

22. McNeil, A.J. and Frey, R. (2000). Estimation of tail-related risk measures for heteroscedastic financial time series: an extreme value approach. Journal of Empirical Finance. 7: 271-300.

23. Muller, N. and Yohai, V.J. (2008). Robust estimates for GARCH models. Journal of Statistical Planning and Inference. 138: 2918 - 2940.

24. Nakatsuma, T. (1998). Markov-Chain Sampling Algorithm for GARCH Models. Studies in Nonlinear Dynamics and Econometrics. 3(2): 107 - 117.

25. Nakatsuma, T. (2000). Bayesian Analysis of ARMA-GARCH Models: A Markov Chain Sampling Approach. Journal of Econometrics. 95(1): 57 - 69.

26. Nelson, D.B. (1991). Conditional Heteroskedasticity in Asset Returns: A New Approach. Econometrica. 59: 347 - 370.

27. Nawaz, F. and Afzal, M. (2011). Value at risk: Evidence from Pakistan Stock Exchange. African Journal of Business Management. 5(17): 7474-7480.

28. Peng L. and Yao Q. (2003). Least absolute deviations estimation for ARCH and GARCH models. Biometrika. 90: 967 - 975.

29. Qayyum, A. and Nawaz, F. (2011). Measuring Financial Risk using Extreme Value Theory: evidence from Pakistan. MPRA Working Paper 29288, University Library of Munich, Germany. 